Guy ACHARD-BAYLE

Université Paul Verlaine, Metz

\title{
SI, DES MARGES AU CCEUR DE LA CONDITION, ET VICE-VERSA. ÉTUDE DE CAS ET RÉFLEXION SUR LA RELATION - ET LE PARCOURS - CENTRE-PÉRIPHÉRIE- CENTRE
}

\section{Introduction}

Je voudrais commencer, dans une première partie, par apporter, au travers de quelques commentaires, ma contribution au débat qui a servi de cadre épistémologique au colloque dont nous lisons ici les actes: la relation centrepériphérie (désormais : RCP); et je le ferai à partir de ma lecture des textes qui nous ont été soumis, extraits des Travaux linguistiques de Prague 2.

Pour ce qui est de ma position personnelle dans le débat, j'y interviens comme sémanticien, mais plus comme sémanticien du discours (j'emploie discours et non texte pour me conformer au métalangage des articles cités) que du système; or dans ces extraits des Travaux linguistiques de Prague 2, la sémantique comme le discours, à l'opposé des formes de l'expression et du système, sont assez peu envisagés.

Pour autant, il est question, dans ces mêmes textes, de «fonction », et plus encore de perspective fonctionnelle de la phrase (functional sentence perspective $^{1}$ ) : l'une et l'autre de ces notions m'encouragent à donner mon point de vue de sémanticien du discours et du texte (il vaudrait d'ailleurs mieux dire : des discours et des textes); il y a également et plus puissamment encore dans ce recueil, l'article de J. V. Neustupný sur vagueness qui m'encourage à participer au débat, même si la notion de vague n'apparaît pas liée au premier abord à la thématique ou à la problématique qui réunissaient les linguistes de Prague dans la revue citée, et qui nous réunissent ici.

Ceci dit, à la traduction de vagueness par vague, je pourrais substituer vaguitude, car ce mot, que je forge sur le modèle de complétude, en est en le pendant, en même temps que le concurrent; je remarque à ce propos que la complétude - référentielle mais aussi syntaxique - préoccupe non seulement les philosophes du langage, mais aussi les linguistes fonctionnalistes. ${ }^{2}$

Dans une deuxième partie, je me consacrerai à une étude de cas, celle de $\mathrm{Si}$ comme subordonnant ou introducteur: de cadre discursif, d'univers de croyance, d'espace mental - ce sont autant d'appellations cognitivo-discursives ou textuelles pour dire, et ceci nous ramène au domaine logico-sémantique évoqué ci-dessus, que la proposition introduite n'est pas une proposition à la valeur de vérité (désormais $\mathrm{VdV}$ ) calculable ou vérifiable.

Autrement dit, Si ouvre un espace conditionnel ou hypothétique certes, mais aussi, discursivement et cognitivement parlant, un espace de débat pour la validation d'une vérité ; où celle-ci s'envisage donc, ou se projette et se négocie, sous condition ou à condition...

\footnotetext{
${ }^{1}$ Suivant DANEŠ (1966 : 18, à la suite de W. Mathesius, 1939 et J. Firbas, 1956, qu'il cite tous deux).

${ }^{2}$ Cf. Clairis (éd. 2005 : 240-241, conférence-débat avec Jean Lallot sur Apollonius Dyscole).
} 
Autrement dit encore, si la VdV d'une conditionnelle n'est pas calculable, i. e. qu'elle n'est précisément pas vériconditionnelle au sens frégéen ou tarskien du terme, ${ }^{3}$ c'est-à-dire vraie (ou fausse) parce qu'elle remplit (ou pas : cf. la question de la complétude) certaines conditions, elle est véridictionnelle au sens où (par exemple, selon CHAROLLES, $2003^{4}$ ) elle dit ou représente à quelles conditions, selon le locuteur, la corrélation des deux propositions peut, ou pourrait être validée (considérée comme, tenue pour vraie).

On voit donc se dessiner ici une conception logico-discursive de la conditionnelle qui prend aujourd'hui le pas en sémantique discursive sur la conception strictement logique de la corrélation ou de l'implication Si $P$, alors $Q$.

Ceci dit, dans les deux approches, logico-sémantique et logico-discursive, cette relation implicative est considérée par nombre d'auteurs comme le cœur, ou « au cœur de la condition», i. e. comme le noyau ou le fondement sémantique de Si. C'est donc la relation ou plus exactement le parcours entre ce noyau ou cœur ou centre conditionnel et sa périphérie ou ses marges «plus ou moins conditionnelles » que je voudrais à la fois présenter et évaluer dans la deuxième partie.

Au-delà de cette étude en deux temps, je voudrais montrer à rebours, c'est-àdire par un mouvement rétroactif de la deuxième à la première partie, qu'il y a du vague dans ce parcours du cœur aux marges, du centre à la périphérie.

$\mathrm{Ou}$ plus exactement je voudrais montrer que s'il y a du vague dans ce parcours, j'en tire plusieurs enseignements suivant QuINE (1960, trad. et rééd. $1999: 188)$ :

i. Qu'il vaut mieux parfois ne pas chercher de remèdes à la présence du flou ; et cela :

ii. Parce que le caractère vague ne trouble pas la $\mathrm{VdV}$ des phrases usuelles où interviennent les mots vagues.

iii. Autrement dit, le vague en question (j'insiste bien sur le fait qu'il s'agit de ce vague-là dont je traiterai), loin de semer le trouble et le désordre dans le système, peut faire la lumière sur celui-ci ;

iv. Ou plus exactement encore, en faisant la lumière sur le centre depuis la périphérie, ce vague-là tendrait à les unifier systématiquement.

J'apporterai, pour clore cette introduction, deux dernières précisions méthodologiques :

- Mes deux parties ne seront pas étanches; je ferai des liens thématiques et problématiques entre elles, dans la mesure où: (i) j'illustrerai ma première partie sur la RCP par des exemples empruntés au système du $\mathrm{Si}$ (plus ou moins) conditionnel; (ii) j'étudierai dans la seconde partie la diversité de ou des $\mathrm{Si}$, et les essais de «réduction» de cette diversité en termes de système et de systématisation.

\footnotetext{
${ }^{3}$ «Si la sémantique est l'étude du sens, la sémantique vériconditionnelle est l'étude du sens en termes de condition de vérité. C'est essentiellement à Frege, puis à Tarski, qu'on doit d'avoir rapproché signification et vérité. Selon eux, comprendre une phrase, c'est connaître les conditions dans lesquelles elle est vraie. Autrement dit, déterminer le sens d'une phrase, c'est être à même de préciser ses conditions de vérité » (BEYSSADE, 2006 : article Vériconditionnel).

${ }^{4}$ Voir aussi ses autres études en bibliographie et l'index de F. Corblin \& H. de Swart (éds, 2004).
} 
- Je laisse de côté la question du continuum (traitée par R. Martin et G. Moignet au début des années 80) entre Si P conditionnel subordonnée et Si P complétive interrogative indirecte (percontative dans le vocabulaire de J. Damourette \& É. Pichon). ${ }^{5}$

\section{Lecture des Travaux linguistiques de Prague 2 et réflexions sur le vague}

Je commence donc par une lecture des Travaux, c'est-à-dire par ce que j'en retiens notamment dans la perspective illustrative qui est la mienne, celle de procéder à une étude de cas.

\subsection{Remarques sur les centres gravitationnels, leur répartition et leur densité (Daneš)}

Je retiens tout d'abord de l'article de F. Daneš, sur «l'universel de langage » que serait la RCP, que l'idée de système au sens de «système régulier et uniforme » est une «idée fausse ». Autrement dit, le système « doit être représenté comme un espace avec une inégale densité d'éléments »; ce que j'interprète, pour ma démonstration, de la manière suivante : le système doit être représenté aussi suivant la densité des éléments répartis inégalement du centre vers la périphérie ou vice-versa ; car, puisqu'on parle d' «espace », je ne veux pas induire de sens unique qui irait du centre vers la périphérie : soit l'idée d'un ordre, et donc d'une norme; ce que ne manquent pas de faire toutefois bon nombre d'auteurs qui se consacrent au $\mathrm{Si}$, on le verra.

Après celle d'inégalité, je retiens de la même citation, la notion de densité : en ce qui concerne $\mathrm{Si}$, il y a en effet des affinités sémantiques qui font qu'il existe des zones plus «chargées» que d'autres: ainsi, si je m'éloigne du centre hypothético-conditionnel vers la périphérie, je constate qu'il y a plus de nuances sémantiques et donc d'occurrences de Si pour marquer la concession, l'opposition (ne serait-ce que par la possibilité qu'il a de s'associer à même où à des coordonnants : et si, mais si...) que pour marquer (i) la restriction, voir :

Un brave homme s'il en fut! (BANYŚ, 2001)

qui est quasiment une forme figée ; ou encore (ii) la justification, voir le Si dit d'énonciation ou austinien :

Il y a de la bière au frigo, si tu en veux. (DUCROT, 1984)

J'ouvre ici une parenthèse pour commenter les usages concessifs : on voit qu'un centre «pèse » d'autant plus dans la chaîne ou le continuum, qu'il est capable de créer des éléments, donc de s'enrichir, d'innover; ainsi dans l'exemple: "Je redoute qu'elles [les élections] se déroulent dans de bonnes conditions, si même elles se tiennent...» (Bernard Kouchner sur France Inter, lundi 11 juillet 2005), on voit apparaitre une forme rare (si même), qui est une conjonction doublement modale ou une double conjonction modale (pour suivre PIOT, 2004), permettant de combiner l'hypothèse à une concession-restriction. Si même n'est évidemment pas même si, ne serait-ce que par la différence qui les sépare en termes statistiques (on y reviendra); il n'empêche, cette conjonction

\footnotetext{
${ }^{5}$ J'ai traité également cette « continuité » dans ACHARD-BAYLE (2007 et 2008).
} 
modalisée prend place dans le continuum qui unit le centre (supposé) du système hypothético-déductif, où trône ou trônerait $S i$, à sa périphérie où se trouverait Même si :

S'il pleut, on ira au cinéma.

Même s'il pleut, on ira à la plage.

On remarquera néanmoins que Si même a du mal à y trouver sa place, tout simplement par défaut d'occurrences, ou faiblesse statistique ; inversement on peut en tirer que, du vague et du flou voire de l'indétermination la plus proche du vide, il ressort, par effet rétroactif, comme en "ombre chinoise », une plus grande netteté des contours des objets ou des éléments qui sont, eux, présents dans le continuum ; je cite ici QUINE (op. cit. : $188^{6}$ ) : «Le vague n'est pas incompatible avec la précision. »

On voit donc, une fois cette parenthèse refermée, et pour revenir à l'argumentation précédente, qu'on soit d'ailleurs au centre ou à la périphérie, qu'il y a çà et là des «centres » [ou des sous-centres] de "gravitation »; ainsi on a (suivant la classification et les exemples de BANYŚ, art. cité) :

- un centre «gravitationnel » au cœur même du système hypothéticoconditionnel qui comprend le potentiel, le contrefactuel, l'implicatif (implication au sens de corrélation envisagée comme possible selon une loi de vérité générale ou plus simplement encore posée comme une exemplification de celle-ci, par exemple selon CORBLIN, 1999);

- un autre centre «gravitationnel » à la périphérie de ce système, par exemple autour de ce que BANYŚ (art. cité) appelle le Si « spécifiant factuel », dont il décline au moins trois variantes (plus deux autres dans des expressions figées ; mais on verra déjà par les registres ou les niveaux de langue des trois premiers exemples, leur caractère déjà périphérique) :

1. Si spécifiant de la prédication principale :

Si elle a vaincu la fière Albion, ce n'est pas par les armes, c'est par son industrie.

2. Si spécifiant l'existence d'une variable saturée de la prédiction principale :

S'il y eut jamais une créature armée pour la résistance, c'est bien celle-là.

3. Si spécifiant de la prédication secondaire :

Si je me souviens bien comment était, jadis, la vie, (je dirais qu') elle était un festin où les cours s'ouvraient, où les vins coulaient.

4. C'est à peine si...

5. C'est merveille si...

Il résulte de cette brève démonstration une première leçon : s'il peut exister dans un système, en l'occurrence un sous-système ou un système particulier du système général, plusieurs centres «gravitationnels », la densité des centres « gravitationnels » n'a pas systématiquement à voir avec le noyau du système particulier lui-même. Autrement dit, pour poursuivre la métaphore «astrophysicienne », et plus précisément pour la poursuivre dans l'esprit des

\footnotetext{
${ }^{6}$ Ch. « Les caprices de la référence $», \S \ll$ Le vague ».
} 
$\mathrm{XVII}^{\mathrm{e} m e}$ et XVIII ${ }^{\text {ème }}$ siècles, s'il y a «pluralités des mondes », il se peut fort bien que le nôtre (autrement dit notre monde référence: ce point de référence qui organise par force tout système) est aussi ou avant tout le résultat d'une systématisation; et ce sont ces «pluralités » que, précisément, des lectures « unificatrices» ou «réunificatrices» tendent à mettre en cause (voir notre deuxième partie).

D'où je tire cette deuxième leçon, méthodologique : la question des centres «gravitationnels » et de leur nombre, et celle de leur propre densité, au-delà de la question du centre et de la périphérie, et de celle du continuum ou de la transition entre ce centre et cette périphérie, sont autant de questions de mises en forme; ou plus exactement pour ce qui nous concerne, en sémantique, de codifications (sémiotisations) ou d'interprétations... ceci dit, sans revendication relativiste!

Mais pour les questions du sens, force est de constater dans la prolifération des interprétations, autrement dit des «nuances», le poids des appréciations parfois plus subjectives qu'intersubjectives ou partagées; donc, force est de constater la légitimité de la revendication d'une simplification, ou d'une « resystématisation » d'un système qui finit par se décomposer ou disparaitre dans la profusion des centres de gravitation et des satellites associés à chacun.

Enfin dernière leçon, toujours méthodologique et épistémologique, en rapport avec ce que je viens de dire de la systématisation, ou de la mise en forme du ou des systèmes : il faut souligner l'importance qui est ou qui doit être accordée à la question de l'intuition dans sa relation, comme je viens de le faire, à la communauté des locuteurs. Je renvoie à ce propos à DANEŠ (art. cité : 17, conclusions sur H. [J.] Kučera ${ }^{7}$ ) :

«It appears that decisions such as those made by Kučera are arbitrary, inasmuch as they lack the necessary systemic motivation. It may be possible, and for purposes of practical application sometimes even necessary, to resort to simplified, unequivocal solutions. But it would be a great, and even dangerous, mistake to suppose that such simplified solutions do justice to the complex reality of language, reality reflected in the linguistic intuition and behaviour of the members of the given language community, both in the synchronistic and in the diachronistic aspects of the facts. If, as is correctly stated also by Kučera, in some instances the native speaker hesitates between two alternative solutions, one obviously has to do with a case of vagueness. Such a case we can, or at least try to, account for by ascertaining all the factors in the play and their mutual relations (clearly, to do so is not easy), but we cannot, and do not want to, eliminate it. It constitutes a part of language and MARTINET $^{8}$ is undoubtedly right in urging that a linguist should not decide in those cases which have been left undecided by language itself. »

\subsection{Le vague : de Neustupný à Quine}

La difficulté avec le vague, et l'article de J. V. Neustupný, vient de ce que cette notion n'est pas a priori une notion linguistique, mais philosophique ou logique. C'est sans doute pourquoi J.V. Neustupný commence par un relevé

\footnotetext{
${ }^{7}$ Sauf erreur de ma part : «Henry Kučera, originally Jindřich » (information trouvée sur Internet, Google + Kučera).

${ }^{8}$ Sans référence.
} 
paraphrastique, qui lui permet et nous permet, si je peux dire, de retomber sur nos pieds de linguistes, puisqu'on y retrouve les notions précédentes: ZentrumPeripherie-Übergang de F. Daneš; et davantage encore pour ce qui nous concerne : «des nuances plutôt que des oppositions » suivant B. Malmberg (cité par F. Daneš, art. cité : 39 - cette référence porte une fois de plus sur la phonologie), ou encore les «border line cases » de L. Bloomfield (1933).

Néanmoins, c'est d'abord sur les questions de logique, tout comme d'ailleurs le signale J. V. Neustupný lui-même dès la deuxième page de son article, que je m'arrêterai dans cette deuxième section consacrée à la notion de vague.

Il y a diverses raisons à ce choix :

1. Le vague est associé à la logique vériconditionnelle de manière contre-exemplaire, si l'on peut dire: en ce qu'il est en fait associé à l'indétermination et aux border line cases de la détermination du vrai ou $\mathrm{du}$ faux; le vague est un contre-exemple du principe du tiers exclu, principium exclusi tertii, dans le calcul de la $\mathrm{VdV}$, autrement dit la recherche et même « la poursuite de la vérité » (suivant le titre de QUINE, 1993).

2. De ce point de vue, deuxième raison, il est utile de répéter avec NEUSTUPNÝ (art. cité : 40) la définition du vague que donne C.S. Pierce, via M. Black, ${ }^{9}$ qui, par parenthèses, est un des auteurs-phares sur la métaphore, autrement dit, sur un domaine qui concerne non pas exactement l'indétermination, mais «l'opacité des contextes dans la donation du référent », (G. Frege) : «A proposition is vague when there are possible states of things. » Or cette définition correspond bien à ce que nous avons dit de la proposition hypothético-conditionnelle ; je paraphrase la suite de la citation de C.S. Pierce (in NEUSTUPNÝ, art. cité : 41) : «Une proposition est vague quand il y a des états de choses possibles. Le locuteur envisage ces états des choses possibles comme pouvant être admis ou exclus. »

Autrement dit, en termes de débat et de délibération, il les considère comme alternativement vrais et non vrais, ce qui est donc en contradiction avec le principe du tiers exclu, mais admis par cette logique (modale) du vague.

Je m'éloigne bien entendu ici de l'utilisation que fait M. Black de C.S. Pierce, et de J. V. Neustupný qui à son tour cite M. Black, puisque les démonstrations portent sur des lexèmes, en l'occurrence sur chair ou sur red (autrement dit sur des questions de prototypicité et de catégorisation); en outre les mêmes auteurs envisagent, alors, la notion de vague comme un outil opératoire, en l'occurrence (avec chair) comme un outil de détermination ou d'assignation, ou encore de complétude référentielle ; or cette opération mobilise des jugements de locuteurs qui, fussent-ils intuitifs, sont recueillis statistiquement et valent donc comme tels (ce qui entre en rapport avec ce que nous avons dit supra de la communauté intersubjective des locuteurs...).

\footnotetext{
${ }^{9}$ Mais sans référence.
} 
Qu'il s'agisse donc de lexèmes n'empêche pas de récupérer ces données : pour ma part j'applique le même «état de choses » indéterminé à un autre niveau de la représentation sémiotique du monde, ou de l'expérience du monde : la proposition, qui, dès lors, devient elle-même dans ces processus de sémiotisation la représentation d'un état d'esprit, ce que nous avons appelé la «véridictionnalité »; nous y reviendrons, car arrivé à ce point, je trouve qu'elle porte un peu mal son nom.

Ceci dit, dans cette acception (ou cette utilisation par transposition que je fais) du vague, du mot à la proposition, je reste comme les auteurs cités au niveau de la détermination du contenu (de l'expression), quand par ailleurs la notion de vague peut être et était utilisée plus haut en termes de systématisation, donc au niveau du système.

Mais cela nous ramène aussi au débat conclusif de la première section : ce serait le vague qui existe ou peut exister dans le contenu même des unités linguistiques ou dans le contenu de ce qu'elles sont censées représenter, qui jetterait le trouble sur et donc créerait du vague dans l'organisation même du système; autrement dit une sorte d'isomorphie régnerait ici entre niveau linguistique et niveau métalinguistique.

3. Dernière raison : le texte de J. V. Neustupný m'a permis de lire ou relire QuINE $\left(1960 / 1999^{10}\right)$, dont J. V. Neustupný dit bien qu'il développe ce vague à partir de matériau linguistique. Ce texte de W. van O. Quine, dont j'ai déjà tiré des conclusions, me permet de passer à la partie proprement consacrée à Si.

\section{Centration, décentration et recentration dans le système hypothético- conditionnel du français}

\subsection{Centre et périphérie : « au cœur et aux marges de la condition » ${ }^{11}$}

L'étude que je propose maintenant est une étude de cas : le cas de Si et de sa diversité ; mais c'est d'abord l'approche du cas qui m'intéressera ici, car, étrangement à force de fixer le centre, l'observation inévitablement part vers les marges!

Si (dans : Si $P, Q$ ou Si $P$, alors $Q$ ) est un cas, tout d'abord, dans la mesure où il n'est qu'une marque parmi d'autres de l'expression de la condition; laquelle marque d'ailleurs peut disparaitre en tant que telle au seul profit des relations sémantiques qui s'instaurent in situ entre les propositions $\mathrm{P}$ et $\mathrm{Q}$. C'est le cas des corrélations hypothético-conditionnelles (i) paratactiques, (ii) interrogatives ou (iii) impératives :

(i) Il entre, je le frappe.

(ii) Entre-t-il, je le frappe.

(iii) Entre, je te frappe. (D'après VoGÜE, 2004 : 86)

Ensuite, en termes notionnels, Si se retrouve aussi bien pour exprimer la condition que pour combiner la condition à l'expression des domaines :

- Du temps : s'il arrive que... si tant est que...

\footnotetext{
${ }^{10} \mathrm{Ch}$. IV, « Les caprices de la référence », $\$ 26:$ «e vague ».

${ }^{11}$ Citation du titre de VogÜe (2004), mais voir aussi SwEETSER (1990).
} 
- De la quantité : une fois que, au cas où...

Cette «hétérogénéité » (VoGÜE, art. cité) lexico-sémantico-syntaxique contraste avec le fondement même de la relation stable qui unit dans tous les cas $\mathrm{P}$ à $\mathrm{Q}$ : toutes les expressions possibles de cette relation logique ou sémantique sont en «quasi synonymie, avec des nuances fines, difficiles à expliciter, volontiers insaisissables » (ibid.). S. de Vogüé se demande ainsi si l'on doit faire la différence entre éventualité vs supposition vs hypothèse, et si, précisément, la condition n'est pas la somme des trois...

J'en profite pour faire une halte ; on revient ici à la problématique que j'ai développée en conclusion de la partie précédente : la diversité sémantique d'une unité lexicale, une fois qu'elle est comprise (saisie et interprétée) contextuellement, ${ }^{12}$ semble à la fois être propre à l'unité (plastique) et résulter du travail de description de l'analyste.

En fait $\mathrm{Si}$ est bien un connecteur d'une telle importance et d'une telle évidence, en ce qu'il est à la source même de toute relation logique (voir ce que l'on a dit plus haut de l'implication), qu'il est applicable comme tel à de nombreuses ou d'innombrables corrélations: on peut alors y voir (i) soit une infinité de nuances qui résultent de la richesse des « expériences corrélatives » que nous faisons des entités et des événements dans notre monde attesté, et davantage encore dans celui que nous créons (supposons) comme un monde possible, parmi les mondes possibles; (ii) soit une sorte de lexème au sémantisme fondamentalement réduit, circonscrit, en même temps qu'assez vague pour se prêter à de multiples utilisations en contexte.

Ainsi, comme le fait VoGÜE $\left(2002^{13}\right)$, on peut à la fois revendiquer l'unité et la diversité, ou encore la «nucléarité » du système, et son éclatement : VOGÜE (ibid.) compare à cet effet Si et l'imparfait ; et l'on trouve la même démarche chez BRES (2005) à propos de l'imparfait et donc de l'imparfait d'hypothèse par rapport à l'imparfait « tout court ».

On peut donc essayer de voir maintenant comment se fait chez VoGÜE (2004) ce passage du centre ou du « cœur » aux «marges », sachant :

que la condition définie ci-dessus est la somme des trois «nuances » : éventualité + supposition + hypothèse ;

que $\mathrm{Si}$ en tant que tel est la seule conjonction apte à figurer dans les trois contextes (vs : «dans l'éventualité où »+ «à supposer que »+ «dans l'hypothèse où ») :

«Les autres constructions permettent d'envisager des éventualités, d'opérer des suppositions, elles ne permettent pas d'émettre des hypothèses (ou du moins, des hypothèses prises comme telles : la locution dans l'hypothèse où prend une hypothèse et la transforme en supposition).

\footnotetext{
12 Par parenthèses: le seul texte des Travaux de linguistique de Prague 2 qui parle un peu du contexte est celui d'O. Leška.

13 «Un, plus que ce par exemple, ou quelque, fait partie de ces unités, comme si, ou peut-être comme l'imparfait, dont la variation est particulièrement importante. Un objectif serait en fait d'arriver à comprendre la façon dont l'ensemble de ces valeurs s'organisent entre elles. »
} 
C'est d'ailleurs une autre raison pour laquelle si fait figure de marqueur prototypique de la condition : non seulement il peut remplacer toutes les autres constructions, mais il a aussi cet emploi [hypothétique] que les autres constructions n'ont pas et qui correspond à la valeur prototypique de la condition. » (VOGÜE, $2004: 101-102$ )

\subsection{Du « cour aux marges »}

2.2.1. Le cour ou le centre concerne (et consiste en) l'expression des lois, des déductions, des inférences; soient :

1. «L'hypothèse pour la forme », expression d'une relation implicative sur la base d'une vérité générale :

Si un nombre est compris entre 0 et 1 , son carré est compris entre 0 et 1 (suivant Frege).

2. La déduction :

S'il appelle, (c'est qu') il a quelque chose à demander.

3. La déduction métalinguistique :

Si c'est un voilier, c'est un bateau.

4. La thématisation ou la reprise :

Il est chez Paul et s'il est chez Paul, il ne peut avoir d'ennuis.

Il fait beau? Eh bien s'il fait beau, on n'a qu'à aller se promener.

5. Les emplois de $\mathrm{Si}$ («fictif») à l'imparfait: contrefactuel et potentiel.

2.2.2. Aux marges : on ne parle plus d'expression de la condition; en effet, on ne peut insérer, d'une manière ou d'une autre, le mot «condition» dans ces énoncés qui expriment (une relation de) ou servent à une :

1. Opposition/restriction :

S'il est riche, cela ne se voit pas.

S'il est riche, [*dans ces conditions] cela ne se voit pas /

S'il est riche, il n'est pas milliardaire.

$S$ 'il est riche, [*dans ces conditions] il n'est pas milliardaire.

2. Explication :

S'il est riche, c'est qu'il a gagné au loto.

S'il est riche, [*dans ces conditions] c'est qu'il a gagné au loto.

VOGÜE (art. cité : 107) parle ainsi d' "élasticité [...] paradoxale» du Si «prototype de la condition» qui s'étend «hors champ», par exemple, à la concession.

Mais, pour finir, S. de Vogüé réunit tous ces emplois dans un même tableau ; ce qui est de nouveau «paradoxal » (VOGÜE, art cité : 10).

Pour ce qui est des énoncés recensés comme étant dans les marges, S. de Voguié se contente de remarquer qu'ils correspondent tous à une «évaluation de $\mathrm{p} \gg$ sans aller plus loin.

Elle aurait pu prolonger la systématisation en termes d'unification, réunification ou recentration des marges. En effet, dans la première partie de son article, en énonciativiste néo-benvenistienne, elle focalise sa réflexion sur le sujet en tant qu'évaluateur, précisément : en dehors du fait que, suivant une première 
distinction, il peut être le sujet soit d'une narration soit d'une argumentation ; et, suivant une seconde distinction :

- ou bien c'est le sujet qui sélectionne $\mathrm{Q}$ en fonction (à cause) de $\mathrm{P}$ :

$V u P$, $S$ sélectionne $Q$;

- ou bien c'est la sélection qui se fait d'elle-même, mais suivant $S$ :

Selon $S$, $P$ sélectionne $Q$.

Mais, dans les deux cas, « $\mathrm{S} »$ intervient :

«Dans les deux cas, il faut que $\mathrm{S}$ ait perçu et évalué [je souligne] p [...] dans le premier cette évaluation justifie et donc précède la sélection où $\mathrm{S}$ témoigne de son libre-arbitre, alors que dans le second les deux opérations ne sont en fait pas dissociées : l'évaluation de p consiste à estimer que p sélectionne q. » (VoGÜE, art. cité : 99-100)

Un pas de plus, et on généralise encore ce système de subjectivisation : de « la sélection de q rapportée au libre arbitre du sujet » à : « une nécessité qui serait attachée à $\mathrm{p}$ (du point de vue du sujet)» (ibid.). La différence est dans «l'étendue » (ibid.) de l'évaluation (partielle vs complète).

\section{3. (Pour finir, sinon conclure) Recentration}

Un dernier pas... et l'on passe à une systématisation «topicaliste» de l'ensemble des valeurs, emplois ou usages de $\mathrm{Si}$.

C'est ce qu'a fait M. Charolles en termes de véridicité (CHAROLLES, art. cité, 2003) à la suite de HAIMAN (1978); on rejoint ici les questions de «syntaxe fonctionnelle » abordées supra.

Ce que j'ai ajouté pour ma part (2006) à l'approche de M. Charolles, c'est une lecture plus dialogique ou polyphonique, mais toujours macro-syntaxique : l'évaluation de la corrélation $\mathrm{P}, \mathrm{Q}$ devient un débat, ${ }^{14}$ où d'une manière ou d'une autre, le point de vue des autres, ou un autre point de vue, donc des discours hétérogènes (extérieurs ou antérieurs) sont convoqués, ou « reconvoqués » (d'où des effets d'écho)...

C'est une recherche qui reste largement à compléter (ACHARD-BAYLE, 2009b).

\section{BIBLIOGRAPHIE}

ACHARD-BAYLE, G. (2006), Si polysémique \& si polyphonique, in Perrin L. (éd.), Le sens et ses voix. Dialogisme et polyphonie en langue et en discours, Université de Metz, CELTED, coll. Recherches linguistiques 28, p. 407-434.

ACHARD-BAYLE, G. (2007), De l'extra- à l'intraprédicatif : polyvalence de Si ?, SKY Journal of Linguistics, en ligne (consulté en juin 2009): [http://www.ling.helsinki.fi/sky/skyjol-en.shtml], p. 9-34.

ACHARD-BAYLE, G. (2008), Si poly... quoi ? Pour un traitement discursif de la diversité des Si, in : O. Bertrand et al. (éds), Discours, diachronie, stylistique du français, Études en hommages à Bernard Combettes, Berne, Peter Lang, p. 55-73.

\footnotetext{
${ }^{14}$ Avec une possible nuance « contrastive » (ACHARD-BAYLE, 2009a).
} 
ACHARD-BAYLE, G. (2009a), Détachement thématique et organisation en écho du texte : le cas des Si $P$ contrastives, in: D. Apothéloz, B. Combettes \& F. Neveu (éds), Les Linguistiques du détachement, Actes du colloque de Nancy, 7-9 juin 2006, Berne, Peter Lang, p. 3-20.

ACHARD-BAYLE, G. (2009b), Comme si... intégrateur(s) d'espaces. Métaphore et condition, espaces mentaux à la puissance 2, communication au colloque AFLICO'3, «Grammars in Construction(s) / Grammaires en Construction(s) », université Paris-Ouest, 27-29 mai 2009.

BANYŚ, W. (2001), Valeurs de vérité, échelle épistémique de fait/non fait et types d'emplois du si propositionnel conditionnel, Studia kognitywne, 4, en ligne (consulté en juin 2009) :

[www.ispan.home.pl/zakjez/semantyka/studiakogn/sk4_banys.pdf].

BlaCK, M. (1949), Language and Philosophy, Ithaca, Cornell University Press.

BloOMFIELD, L. (1933), Language, New York, Henry Holt.

BEYSSADE, C. (2006), Vériconditionnel, in D. Godard, L. Roussarie et F. Corblin (éds), Sémanticlopédie : dictionnaire de sémantique en ligne (consulté en juin 2009) : [http://www.semantique-gdr.net/dico/].

BRES, J. (2005), L'imparfait: l'un et/ou le multiple ? À propos des imparfaits « narratif » et «d'hypothèse », Cahiers Chronos, 14, p. 1-32.

Charolles, M. (1997, rééd. 2004), L'encadrement du discours. Univers, champs, domaines et espaces, nouvelle version en ligne :

[www.lattice.cnrs.fr/IMG/pdf/cadresdisc.pdf].

Charolles, M. 2003, De la topicalité des adverbiaux détachés en tête de phrase, Travaux de Linguistique, 47, p. 11-51.

Charolles, M. \& PrÉvost, S. (éds., 2003), Adverbiaux et topiques, Travaux de Linguistique, 47.

Charolles, M. \& PÉRY-WoOdley, M.-P. (éds., 2005), Les adverbiaux cadratifs, Langue française, 148.

ClaIRIS Ch. (éd., 2005), Travaux de linguistique fonctionnelle 1992-2002, Paris, L'Harmattan.

CORBLIN, F. (1999), Une approche cognitive des conditionnelles : hypothèse et monotonie, LINX 41, p. 21-38.

Corblin, F. \& DE SwART, H. (éds., 2004), Handbook of French Semantics, Stanford, CSLI (Center for the Study of Language and Information) Publications.

DAmouretTE, J. \& PiCHON, É. (1936), Des mots à la pensée. Essai de grammaire de la langue française, Tome 5, Paris, D’Artrey.

DANEŠ, F. (1962), [cité par NEUSTUPNÝ, 1966], Zusammenfassung des Diskussionsbeitrages, Zeichen und System der Sprache II, Berlin, Akademie Verlag.

DANEŠ, F. (1966), The Relation of Centre and Periphery as a Language Universal, Travaux linguistiques de Prague 2, p. 9-20.

DuCRot, O. (1984), Le dire et le dit, Paris, Les Éditions de Minuit.

FIRBAS, J. (1966) [cité par DANEŠ 1966], Non-thematic subjects in contemporary English, Travaux Linguistiques de Prague 2, p. 239-256. 
FREGE, G. (1892 / 1971), Ueber Sinn und Bedeutung, Zeitschrift für Philosophie und philosophische Kritik, trad. fr. in : Écrits logiques et philosophiques, Paris, Éditions du Seuil, p.102-126.

HAIMAN, J. (1978), Conditionals are topics, Language 54, p. 564-589.

KUČERA, C. (1961) [cité par DANEŠ 1966], The Phonology of Czech, The Hague, Mouton \& Co.

LEŠKA, O. (1966), «Le centre » et «la périphérie » des différents niveaux de la structure linguistique, Travaux linguistiques de Prague 2, p. 53-57.

LYCAN, W. (2001), Real Conditionals, Oxford, Clarendon Press.

MALMBERG, B. (1940), Observations sur le système vocalique du français, Acta linguistica II, p. 232-246.

MALMBERG, B. (1942 / 1943), À propos du système phonologique de l'italien, Acta linguistica III, p. 34-43.

Martin, R. (1983), Pour une logique du sens, Paris, Presses universitaires de France.

MATHESIUS, V. (1939) [cité par DANEŠ 1966], On the so-called Functional Sentence Perspective, SaS 5, p. 171-174.

MoIGNET, G. (1981), Systématique de la langue française, Paris, Klincksieck.

NEUSTUPNÝ, J. V. (1966), On the Analysis of Linguistic Vagueness, in Travaux linguistes de Prague 2, p. 39-51.

PIOT, M. (2004), La conjonction même si n'existe pas !, in : Ch. Leclère et al. (éds), Lexique, Syntaxe et Lexique-Grammaire, Benjamins, Amsterdam, p. 481-491.

QUINE, W. VAN O. (1960, trad. fr.1977, rééd. 1999), Le Mot et la chose, Paris, Flammarion, coll. Champs.

Quine, W. van O. (1993), La poursuite de la vérité, Paris, Éditions du Seuil.

SWEETSER, E. (1990 / Paperback $8^{\text {th }}$ reprint 1998), From etymology to pragmatics. Metaphorical and cultural aspects of semantic structure, Cambridge, Cambridge University Press.

TRAVAUX LINGUISTIQUES DE PRAGUE 2, Les problèmes du centre et de la périphérie du système de la langue, Prague, Academia-Éditions de l'Académie Tchécoslovaque des Sciences, 1966.

VoGÜE, S. (DE) (2002), L'article un, la position sujet, et la relation avec le prédicat, Actes du colloque Indéfinis et prédications, Paris Sorbonne, 3-5 octobre 2002, article disponible en ligne (consulté en juin 2009): [http://www.llf.cnrs.fr/Gens/Vogue/article_un_sdv.pdf].

VoGÜE, S. (2004), Si, au centre et aux marges de la condition, in : C. Hare (éd.), L'Hypothèse au miroir des langues, Paris, L'Harmattan, p. 85-117. 


\section{SUMMARY}

In this paper, I deal with the polysemy of the conditional conjunction $S i$ (If), which has been considered by several authors as "more or less" conditional (Lycan, Sweetser, Vogüé), that is to say, as a central (logical) vs. a marginal (discursive) marker of conditionality. Following the concept of vagueness, introduced by Neustupný in the works of the Prague Linguistic Circle (Travaux linguistiques de Prague 2), I first want reconsider these supposed degrees of conditionality. Then, following the Functional Sentence Perspective developped by Mathesius, Firbas, Daneš, and the Discursive Frames Theory developed more recently by Charolles following Fauconnier (espaces mentaux - mental spaces) and Martin (univers de croyances - belief universes), I want to reconsider the both semantic and discursive question of the central vs. marginal conditionality of Si, and to complete or to reverse this perspective: from centre to margins... and vice versa. 